\title{
Fourteen-year study of hospital admissions for diverticular disease in Ontario
}

\author{
Eiran Warner $\mathrm{BSc}^{1}$, Eric J Crighton $\mathrm{PhD}^{1}$, Rahim Moineddin $\mathrm{PhD}^{2}$, \\ Muhammad Mamdani PharmD MPH ${ }^{3,4}$, Ross Upshur MA MD MSc CCFP FRCPC 1,2,3
}

E Warner, EJ Crighton, R Moineddin, M Mamdani, R Upshur. Fourteen-year study of hospital admissions for diverticular disease in Ontario. Can J Gastroenterol 2007;21(2):97-99.

BACKGROUND: Diverticular disease is one of the most common gastrointestinal conditions affecting the Canadian population, yet very little is known about its epidemiology.

OBJECTIVE: The aim of the present study was to measure the rate of hospital admission for diverticular disease by age and sex over a 14-year period in the population of Ontario.

PATIENTS AND METHODS: The present study was a retrospective, population-based cohort study of all hospital admissions for diverticular disease from 1988 to 2002.

RESULTS: There were 133,875 hospital admissions during the period. Admission rates increased with age, and women were admitted at higher rates than men across all age groups.

CONCLUSION: Diverticular disease is an important cause of gastrointestinal morbidity. As the population ages, a rise in the incidence of diverticular disease can be anticipated. Future studies to explain sex difference in admissions are required.

\section{Étude d'une durée de quatorze ans sur les hospitalisations causées par une diverticulite}

Key Words: Diverticulitis; Diverticulosis; Epidemiology

In most industrialized countries, diverticulosis is believed to affect $30 \%$ to $50 \%$ of the population over 60 years of age, with $10 \%$ to $25 \%$ of cases developing complications such as diverticulitis and diverticular hemorrhage (1). Diverticulosis is thought to be caused by the combination of an increase in intraluminal pressure and weakening of the colonic wall (2), both of which are exacerbated by aging, and a low-fibre diet $(3,4)$. Although not typically life-threatening if treated, diverticular disease causes significant pain and suffering for those afflicted, and places a substantial burden on the health care system

Studies examining trends in diverticular disease hospitalizations are limited. A single study in England (5) found that hospitalization rates increased over the time period of 1989 to 2000, with male rates being higher for patients younger than 50 years of age, and female rates higher for patients older than 50 years of age. The study, however, did not examine seasonal variations in the complications of diverticular disease, a potentially important clue to its pathogenesis. Seasonality of occurence is a conspicuous feature of many gastrointestinal diseases such as peptic ulcer disease. This may be related to the seasonal incidence of infectious agents or dietary habits. Therefore, we tested the hypothesis that seasonal variation for diverticulitis hospitalizations may exist. There have been no large-scale epidemiological studies of hospitalization patterns for diverticular disease in North America.

To address this gap, the present study examined hospitalization patterns for diverticular disease in Ontario by age and sex over a 14-year period to answer the following questions: Do hospitalization rates vary by age and sex? How do rates vary over the 14-year period? Are there seasonal patterns to these hospitalizations?

\section{METHODS}

A retrospective population-based cohort study design was used to examine temporal patterns of hospital admissions for diverticular disease between April 1, 1988, and March 31, 2002, in Ontario. Admission data were obtained from the Canadian Institute for Health Information - Discharge Abstract Database for the principal diagnosis of diverticular disease (World Health Organization's International Classification of Diseases - Ninth Revision code 562). This database records discharges from all acute care hospitals in Canada, documenting a scrambled patient identifier; date of admission and discharge; up to 16 diagnoses as coded by the International Classification of Diseases Ninth Revision - Clinical Modification; and up to 10 procedures.

${ }^{1}$ Primary Care Research Unit, Sunnybrook Health Sciences Centre; ${ }^{2}$ Department of Family and Community Medicine; ${ }^{3}$ Institute of Clinical Evaluative Sciences; ${ }^{4}$ Health Policy Management and Evaluation, University of Toronto, Toronto, Ontario

Correspondence: Dr Ross Upshur, Sunnybrook Health Sciences Centre, 2075 Bayview Avenue, Room E-349, Toronto, Ontario M4N 3M5.

Telephone 416-480-6100 ext 1691, fax 416-480-4536, e-mail ross.upshur@sw.ca

Received for publication November 8, 2005. Accepted June 22, 2006 


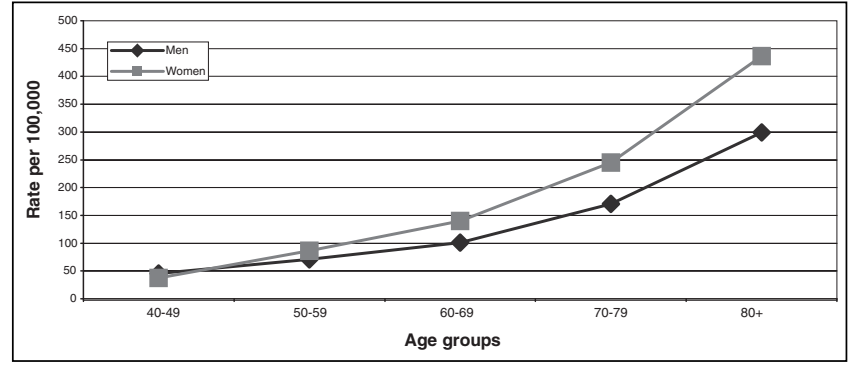

Figure 1) Age-specific hospital admission rates for diverticular disease (average: 14-year period)

Research has shown that the diagnoses in this database have a high degree of accuracy, with less than $1 \%$ of the basic information missing (6).

All hospital discharges for diverticular disease were extracted by age and sex for each month over the study period. Annual census data for each age group for residents of Ontario were obtained from Statistics Canada. Monthly population estimates were derived through linear interpolation. These data were then used to calculate monthly hospitalization rates for patients older than 40 years of age, standardized for month length. Transfers from one acute care hospital to another within this study group were not included in the analysis.

Several time series analysis techniques were used. First, spectral analysis, which detects periodicity in time series, was conducted (7). The data were detrended before conducting spectral analysis using moving averages. Two tests - the Fisher's Kappa (FK) test and the Bartlett Kolmogorov Smirnov (BKS) test - were conducted for the null hypotheses that the series was strictly white noise. The FK test is designed to detect one major sinusoidal component buried in white noise, while the BKS test accumulates departures from the white noise hypotheses over all frequencies (8). Following this, the autocorrelation function was used to measure the correlation between observations at different time lags (9). A strong correlation between the observations at 12 time lags indicates a strong seasonality of the period 12 . Finally, $\mathrm{R}^{2}$ autoregression coefficients $\left(\mathrm{R}^{2}\right.$ Autoreg $)$ were calculated to quantify the strength of the seasonality (10). The $\mathrm{R}^{2}$ Autoreg is interpreted the same way as the coefficient of determination in classic regression: values from 0 to less than 0.4 represent nonexistent to weak seasonality, 0.4 to less than 0.7 represent moderate to strong seasonality, and 0.7 to 1 represent strong to perfect seasonality. All statistical analyses were performed using SAS version 8.2 (SAS Institute Inc, USA).

\section{RESULTS}

Between fiscal years 1988 and 2001, there were a total of 133,875 hospitalizations in Ontario for diverticular disease in patients older than 40 years of age. Over this time period, rates increased from 94 per 100,000 in 1988 to 105 per 100,000 in 2001.

Admission rates were higher for women than men in almost all age groups and this difference increased with age. One exception was the 40- to 49-year age group, where admission rates for men were approximately $21 \%$ higher than for women In the 50- to 59-year age group, women had a 15 per 100,000 higher rate than men but by the 80 -year and older age group, this rate difference had increased to 137 per 100,000 . For both men and women, rates increased with age. Men had a rate of 45 per 100,000 in the 40- to 49-year age group which increased

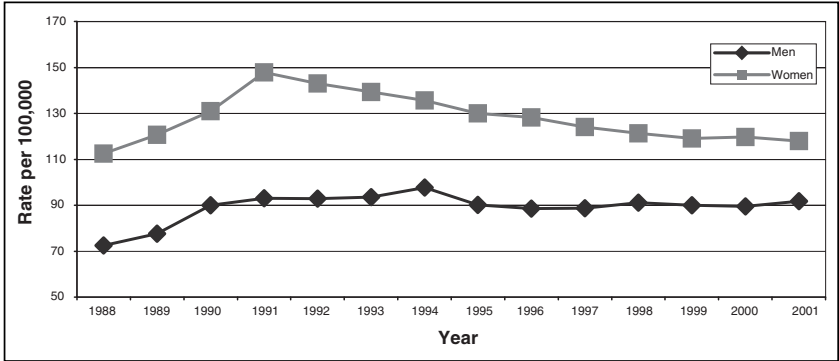

Figure 2) Admission rates for diverticular disease from 1988 to 2001 for patients older than 40 years of age

to 299 per 100,000 in the 80 -year and older age group. Women had an admission rate of 38 per 100,000 in the 40- to 49-year age group which increased to 436 per 100,000 in the 80 -year and older age group (Figure 1).

Hospitalization rates for women increased from 112 per 100,000 in 1988 to a rate of 147 per 100,000 in 1991 (Figure 2) and then gradually decreased to approximately 119 per 100,000 in 2001. Hospitalization rates for men increased from 73 per 100,000 in 1989 to 93 per 100,000 in 1991 . This rate remained more or less constant until 2001.

The FK and BKS tests for seasonality yielded inconsistent results when the analysis was broken down by either sex or age. Although the aggregate $\mathrm{R}^{2}$ Autoreg value indicated moderately strong seasonality, the individual values showed only weak seasonality.

\section{DISCUSSION}

The present study examined trends in hospitalization rates for diverticular disease over a 14-year period. Results revealed that women typically had higher hospitalization rates than men; these differences increased with age in both sexes. Between 1988 and 1992, hospitalization rates increased substantially for both sexes. For women, these rates declined over much of the remainder of the study period, while for men, the rates remained relatively stable. There was very little evidence of seasonality in admission rates for diverticular disease.

The pattern of higher rates in men younger than 50 years of age, and higher rates in women older than 50 years of age, is consistent with findings from previous studies $(5,11,12)$. Suggested explanations for these sex differences include the positive effect for men that testosterone may have on protecting the wall of the colon from weakening with age (13), and the negative effect for women that pregnancy may have on decreasing intestinal motility and weakening of the colon wall due to high pelvic pressures generated during labour (14). Further research is needed to support or refute these hypotheses.

The increase in hospitalization rates found in our study, between 1988 and 2001 are proportionately higher in men but lower in women than the changes identified by Kang et al (5). They observed an overall increase from 25.1 to 28.2 per 100,000 in England between 1989 and 2000. Their lower absolute rates may be explained by their use of the whole population as their denominator rather than the population older than 40 years of age. Also, barium enema examinations and colonoscopies may have been more prevalent in Ontario hospitals for patients presenting with abdominal symptoms, leading to higher ascertainment. Nevertheless, true prevalence differences may exist between Canada and the United Kingdom. 
The rate increase observed in our study between 1988 and 1992 is similar to the upward trends reported during this time period in Ontario for other health conditions including myocardial infarction, chronic obstructive pulmonary disease and congestive heart failure, and overall hospitalizations. Further research is required to explain these findings.

Because seasonality is well-documented in peptic ulcer disease, which has a bacterial etiology, it would be conceivable that seasonal variation for diverticulitis may exist as well; particularly if seasonal dietary patterns or infectious agents were implicated in triggering illness sufficient to warrant hospitalization. To the best of our knowledge, no previous study of diverticular disease has checked for such variation. However, we found no evidence of seasonality in our study. Over the past 20 years, abundant fruits and vegetables have been available year round to Canadian consumers. The absence of seasonality in the present study does not rule out the possibility that diet plays an important role in the complication rate of diverticular disease.

The chief limitation in the present study is the inability to separate out distinct types of diverticular disease. We aggregated

\section{REFERENCES}

1. Stollman N, Raskin JB. Diverticular disease of the colon. Lancet 2004;363:631-9.

2. Ferzoco LB, Raptopoulos V, Silen W. Acute diverticulosis. N Engl J Med 1998;338:1521-6.

3. Painter NS, Burkitt DP. Diverticular disease of the colon: A deficiency disease of Western civilization. Br Med J 1971;2:450-4.

4. Mimura T, Bateman AC, Lee RL, et al. Up-regulation of collagen and tissue inhibitors of matrix metalloproteinase in colonic diverticular disease. Dis Colon Rectum 2004;47:371-8;378-9.

5. Kang JY, Hoare J, Tinto A, et al. Diverticular disease of the colon - on the rise: A study of hospital admissions in England between 1989/1990 and 1999/2000. Aliment Pharmacol Ther 2003;17:1189-95.

6. Williams J, Young W. Appendix - A summary of studies on the quality of health care administrative databases in Canada. Patterns of Healthcare in Ontario. The Ices Practice Atlas: Canadian Medical Association, 1996. codes to examine the overall impact of diverticular disease. Another limitation of our study is that changes in the rates of usage of other health services for diverticular disease, such as outpatient physician visits, could not be assessed. Also, surgery rates and mortality statistics were not included. The strength of the present study lies in its longitudinal base and large population size, combined with the use of a comprehensive timeseries analysis approach applied to age and sex.

With Ontario's aging population, hospitals will almost certainly experience an increase in diverticular disease rates. The present study increases our understanding of the pattern of this illness and can be expected to inform the development of age- and sex-targeted preventive strategies, as well as more effective allocation of health care resources.

ACKNOWLEDGEMENTS AND AFFILIATIONS: The present research was supported by an operating grant from the Canadian Institutes of Health Research (grant \#MOP57928). The authors thank Shari Gruman for her expert assistance in formatting the manuscript.

7. Fuller WA. Introduction to Statistical Time Series. New York: John Wiley and Sons Inc, 1976.

8. Priestly MB. Spectral Analysis and Time Series. New York: John Wiley and Sons Inc, 1976.

9. Box G, Jenkins GM. Time Series Analysis: Forecasting \& Control, 2nd edn. San Francisco: Holden-Day, 1976.

10. Moineddin R, Upshur RE, Crighton E, Mamdani M. Autoregression as a means of assessing the strength of seasonality in a time series. Popul Health Metr 2003;1:10.

11. Parks TG. Natural history of diverticular disease of the colon. A review of 521 cases. Br Med J 1969;4:639-42.

12. Kyle J, Davidson AI. The changing pattern of hospital admissions for divertical disease of the colon. Br J Surg 1975;62:537-41.

13. Delmas PD, Chapunlat RD. Osteoporosis. In: Degroot LJ, Jameson LJ, eds. Endocrinology, 4th edn. Philadelphia: WB Saunders Co, 2001:1244-58.

14. Katz VL. Prenatal care. In: Scott James R, ed. Danforth's Obstetrics and Gynaecology, 9th edn. Philadelphia: Lippincott Williams \& Wilkins, 2003:1-20. 


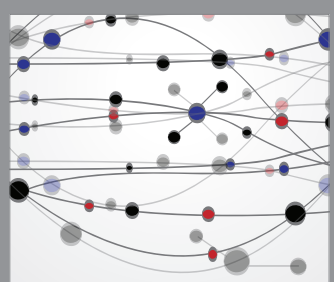

The Scientific World Journal
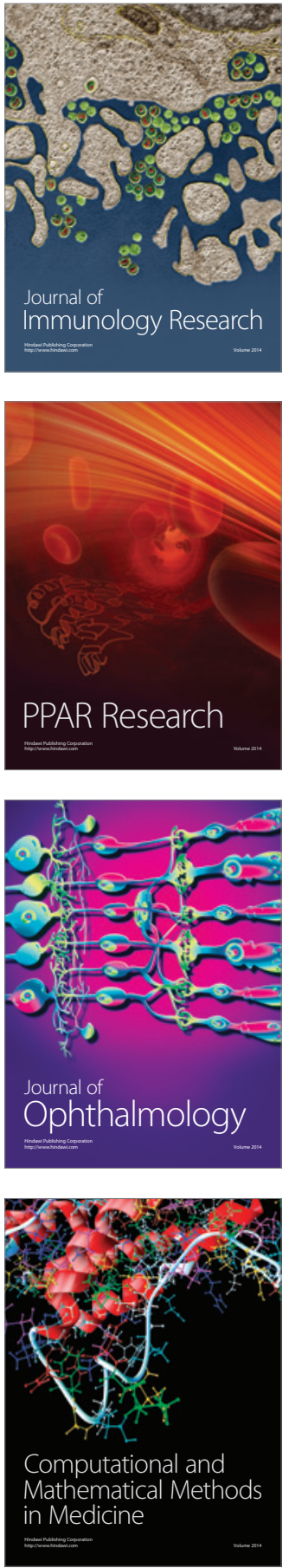

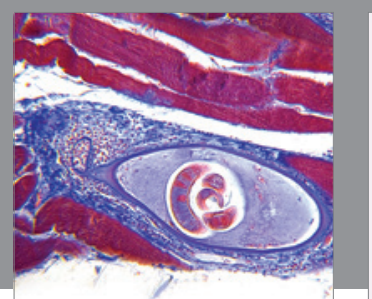

Gastroenterology Research and Practice

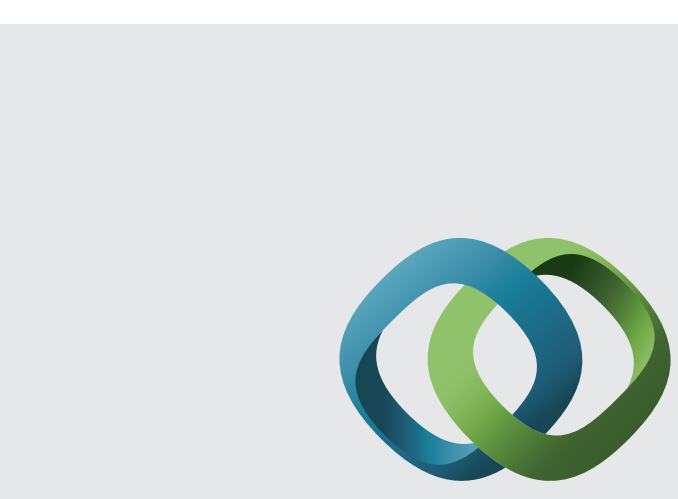

\section{Hindawi}

Submit your manuscripts at

http://www.hindawi.com
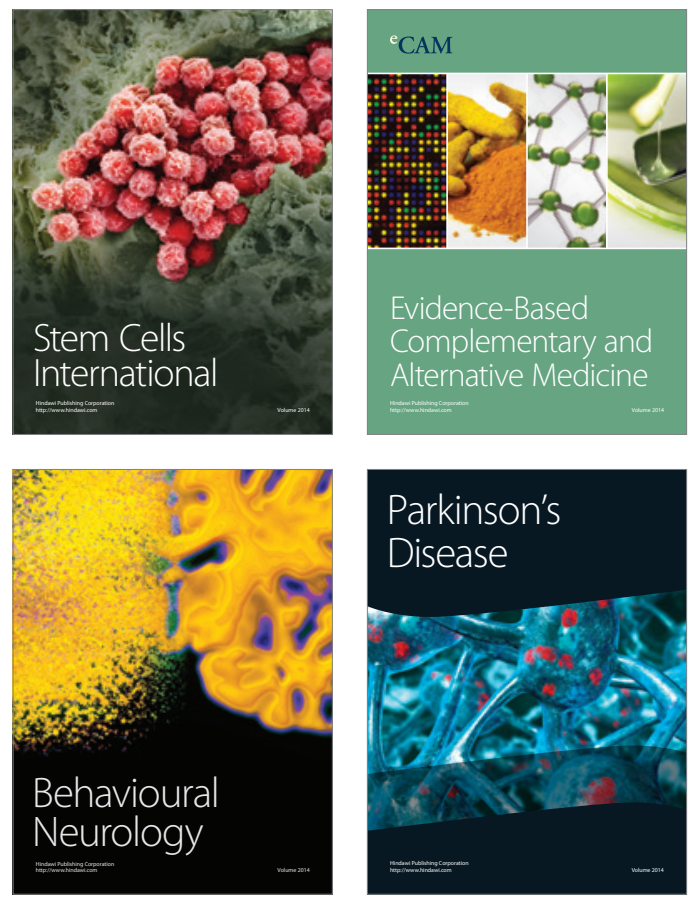
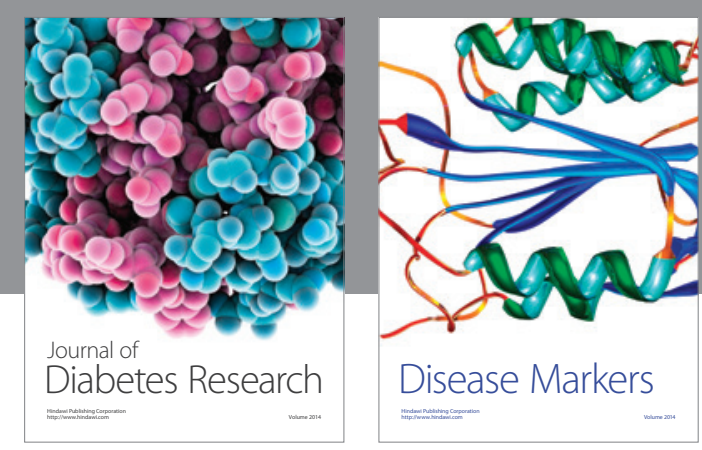

Disease Markers
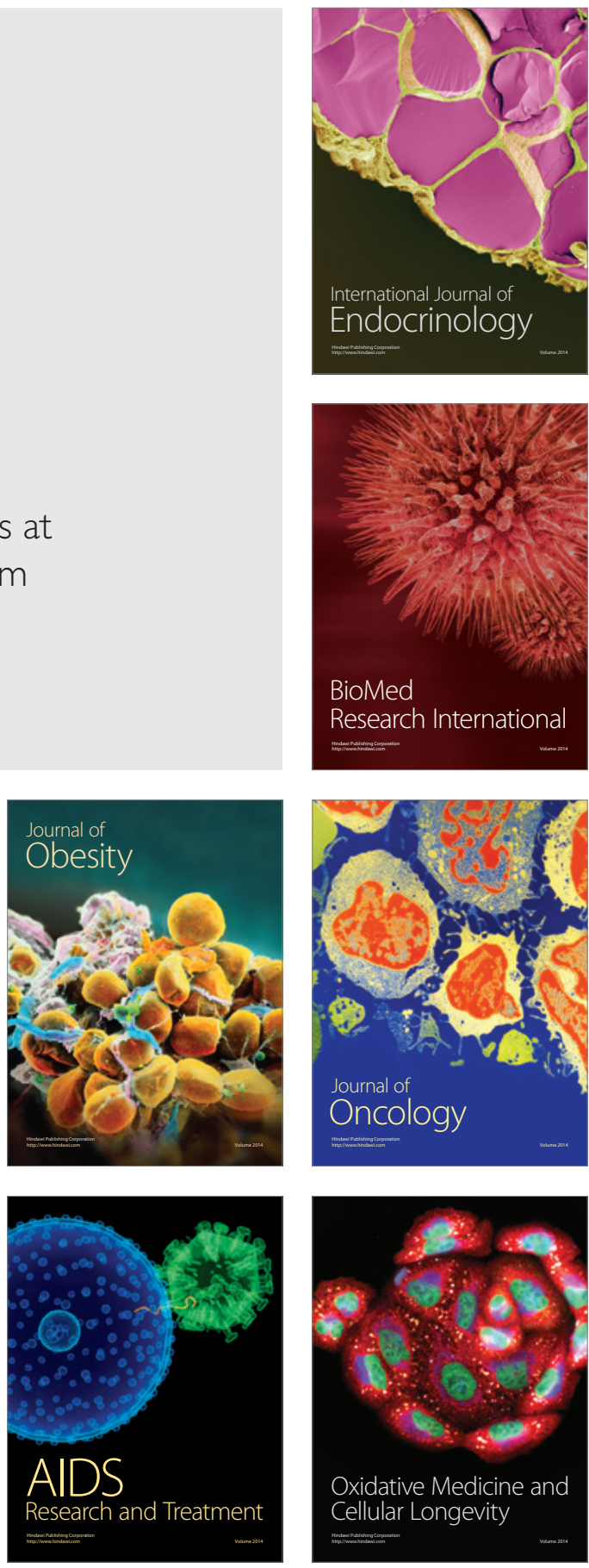